\title{
Bder Adults
}

\author{
Jane E. Myers and Melanie C. Harper
}

Older persons constitute an increasingly large population with significant mental health challenges. The authors review outcome research for both diagnosable conditions and late-life transitions as a basis for evidence-based practice with this population. Implications for clinical practice in professional counseling, counselor education and supervision, and research are considered.

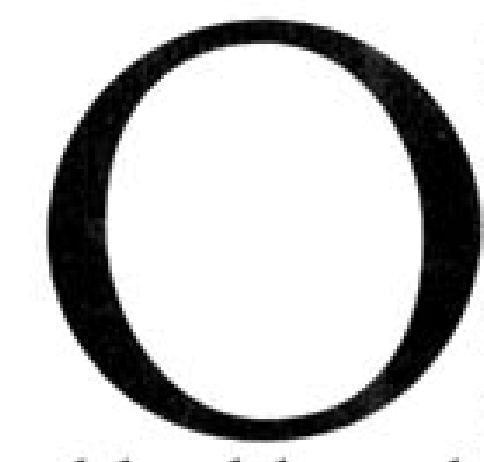

lder adults (persons over age 65) constitute a unique population that has been underserved in the area of counseling services (Myers \& Schwiebert, 1996). An estimated one third of individuals in this population have mental health problems for which professional intervention is needed, including anxiety, depression, and other diagnosable mental disorders (Smyer \& Qualls, 1999), but this estimate fails to incorporate normative developmental issues such as the transition to retirement, grandparenthood, and second careers. When developmental issues are included, clearly the incidence of mental health needs among the older population increases. Given the changes in the demographic structure of society in the United States, specifically the dramatic increase in the numbers of older persons, it is highly likely that professional counselors will encounter older persons and their families as clients (Myers, 1995). As a consequence, counselors' repertoire of knowledge and skills should include strategies for assessment and intervention to address both the common mental health concerns of persons in later life and the normative issues of aging.

Although persons over the age of 60 make up almost 13\% of the total United States population (AARP, 2000), they represent only $6 \%$ to $8 \%$ of persons seen in community mental health clinics and outpatient mental health settings and an even smaller percentage of clients seen by private counseling practitioners (Smyer \& Qualls, 1999). Barriers to the use of these services by this population include bias toward older persons among service providers and reluctance of older persons to seek counseling, as well as a lack of adequate training and supervised clinical practice with this population that would allow counselors to be both comfortable with and effective in service to older clients (Nordhus \& VandenBos, 1998). Such training requires an understanding of effective intervention practices.
In this article, we provide a brief overview of the older population and describe several important differences in counseling with older and younger persons. We review and discuss outcome studies that reveal effective strategies and best practices for counseling with older persons. We accessed these studies from reviews of PsycINFO, the ERIC database, and counseling journals. We also consider implications for clinical practice, counselor training, and needed research.

\section{THE OLDER POPULATION: DEMOGRAPHICS, COUNSELING NEEDS, AND COUNSELING CHALLENGES}

Professional counselors working with older persons need to understand how those in this population are like persons of other ages and how they differ. A brief review of key demographic indicators provides information on some risk factors in later life, such as gender, ethnicity, and income. Commonly experienced circumstances in later life create counseling needs, and the higher incidence of mental health concerns combined with these needs contributes to challenges for counselors working with older clients. It is important to note that, although traditional, stereotypical views of aging present a negative perspective dominated by poor health, disability, functional limitations, and increasing mental illness, recent research on later life reveals that older persons typically age well (Myers, 2003); are resilient in responding to stress, transitions, and change (Myers \& Schwiebert, 1996); and experience a lower incidence of mental illness than do persons of younger ages (Smyer \& Qualls, 1999).

\section{Demographics}

The total population of older persons in the United States numbered 34.5 million in 1999, representing 1 in every 8 persons-an 11 -fold increase during the twentieth century

Jane E. Myers and Melanie C. Harper, Department of Counseling and Educational Development, University of North Carolina at Greensboro. Correspondence concerning this article should be addressed to Jane E. Myers, Department of Counseling \& Educational Development, PO Box 26171, University of North Carolina at Greensboro, Greensboro, NC 27402-6171 (e-mail: jemyers@uncg.edu). 
(AARP, 2000). Life expectancy increased from 47 years in 1900 to 76.7 years in 1998 . The total older population is expected to increase to $20 \%$ of the population by 2030 , with significant increases in older minority populations. Ethnic minorities represented $16.1 \%$ of the older population in 1999 and will increase by $81 \%$ to over $25 \%$ of the older population by 2030 (AARP, 2000).

These population parameters define some of the characteristics of the older population as a whole, but they obscure significant within-group variation that places many older persons at risk. For example, a gender gap in life expectancy results from women living an average of 19.2 years after age 65 , while men live an average of only 16 more years. The ratio of older women to older men is 141:100, with four times as many widows as widowers in later life. In addition, $8 \%$ of older persons are divorced, a significant increase in the last decade (AARP, 2000). Divorced older persons are especially likely to be among the $10 \%$ of older persons who are poor. In fact, the poverty rate for older women continues to be higher than that for older men, with some $20 \%$ of older persons being poor or near poor. Older persons who are members of ethnic minorities are more likely to live at or near the federal poverty level. (The 2001 guidelines for the federal poverty level range from $\$ 8,500$ for a family of one to $\$ 29,730$ for a family unit of eight.)

\section{Counseling Needs and Counseling Challenges}

Common life experiences and transitions in later life often create specific needs for counseling. These transitions include, but are not limited to, coping with loss (e.g., death of spouse or friends, loss of health or disability), adjusting to retirement and reduced income, grandparenthood, second careers, and creating satisfying leisure lifestyles (Santrock, 2002). Each transition can be for better or for worse, and coping strategies that have evolved over a lifetime may mitigate individual circumstances with consequent positive outcomes. On the other hand, older persons are more likely than persons of any other age to experience multiple losses simultaneously, and their capacity to cope with these losses can be significantly compromised, resulting in needs for counseling interventions (Smyer \& Qualls, 1999). Counselors typically are younger than their older clients and need to consider the impact of differences in age and life experience on the counseling relationship. Building rapport may take longer because of the possibility that older persons may be reluctant to seek counseling, may lack familiarity with counseling processes, and may lack experience in discussing or dealing with feelings (Myers \& Schwiebert, 1996). Physical limitations may result in a need to modify the length of counseling sessions and/or assessment procedures (e.g., use of standardized assessment measures). Both individual and group counseling may be needed and may be focused on specific topics or transitions. The tendency for older persons to view counselors in the same manner as physicians (i.e., hierarchical, directive relationship) can be a significant barrier to successful interventions.

Overall, older persons are more similar to persons of other ages than they are different from them, and the full range of counseling skills will be required to meet their needs (Myers \& Schwiebert, 1996). At the same time, increased incidence of depression in response to loss and comorbidity of mental health and physical problems create unique needs for differential diagnosis and treatment (Smyer \& Qualls, 1999). Psychogenic stress can lead to physical health problems, and the reverse can also occur, creating significant comorbidity and complexity of issues in later life (Nordhus \& VandenBos, 1998). These problems tend to occur most often among the middle- and older-old population, those persons ages 75 years and older. Young-old adults, those ages 50 to 75, tend to share more common concerns with younger persons, although the dynamics of the aging process complicate treatment planning even for those older adults, regardless of age, who remain physically healthy.

\section{STUDIES OF COUNSELING EFFECTIVENESS WITH OLDER PERSONS: DIAGNOSABLE DISORDERS}

In 1996, Roth and Fonagy published a comprehensive review of evidence-based effective psychotherapy practices for diagnosable disorders as detailed in English-language journals (Conte, 1998). In the following sections, we summarize their research concerning effective practices with older adults and add findings from more recent empirical studies. The literature is presented in terms of older adults' use of mental health services, general outcome studies with older adults, and effectiveness of specific nonpharmacological interventions with older adults to treat identified mental health problems. In this section, we consider significant mental health issues and diagnosable conditions, based on the traditional medical model for assessment and treatment. In contrast, we address common transitions and life challenges experienced by the majority of or, in some cases, all older persons, conceptualized from a developmental, wellness paradigm in a subsequent section.

\section{Studies of Service Use}

Studies of older adults' use of mental health services, first published in the 1990s, consistently reveal a pattern of under service, both in community mental health and private practice settings (Smyer \& Qualls, 1999). For example, a survey of Los Angeles County psychologists listed in the National Register of Health Service Providers in Psychology (Council for the National Register of Health Providers in Psychology, 1985) concerning services they offered to older adults revealed that, among 114 respondents, only $3.1 \%$ to $4.4 \%$ specialized in serving older adults. Although this study was limited to services available in one urban county and thus cannot be generalized to the older population as a whole, the results are consistent with earlier studies demonstrating a lack of needed mental health services for the older population nationally (Myers \& Schwiebert, 1996). Moreover, the results cannot be generalized across all mental health disciplines, particularly counseling.

Although Roth and Fonagy (1996, p. 323) provided only one survey from the British Department of Health, pub- 
lished in 1994 regarding initial contacts of clients 65 years of age and older with British clinical psychologists, as evidence to support the assumption that older adults receive inequitable access to psychological treatment, they identified several reasons for apparent underutilization of psychological services. These reasons include reluctance by therapists to treat older adults; lack of identification of older adult psychological problems by primary care physicians; and incorrect diagnosis of depression as dementia, which results in a low rate of referral for psychological treatment.

Black, Rabins, German, McGuire, and Roca (1997) noted that older adults' underutilization of psychological services might be related to unrecognized or unacknowledged mental health problems in this population and their lack of Medicare insurance or Medicaid. Black et al. used interviews and assessment instruments to study the use of psychological services and the need for these services among 371 older residents living in six public housing developments in Baltimore, Maryland. The majority of the participants lived alone (95\%), were African American (95\%), and were female (84\%). Of these participants, $33 \%$ indicated they had used mental health services, but only $4 \%$ indicated they had received these services from a mental health specialist. Most of the participants who reported receiving mental health services said that they received these services from a primary medical care provider. More than half the participants who were identified through the interviews and instruments as being in need of mental health services indicated that they had not received mental health services of any kind in the previous 6 months.

Black et al. (1997) found that few of the participants who seemed to have psychiatric disorders rated their mental health status as "poor/very bad," possibly indicating that many were unaware of their need for mental health services. The authors also found a strong correlation between having Medicare insurance and the use of mental health services. Having Medicaid coverage correlated with the use of mental health services among the noncognitively impaired participants. Results from this study indicated that the awareness of a mental health problem and having insurance that covers mental health services are factors in the use of services by some older adults.

\section{General Outcome Studies}

General outcome studies are designed to answer the question, Does counseling work with older adults? Roth and Fonagy (1996) cited several studies that substantiated the efficacy of interventions with older adults. These studies found evidence to suggest that, although older adults respond to counseling as well as or better than younger adults, older adults might require more sessions and a longer involvement in therapy. The increased length of treatment is related to the more numerous life experiences of older adults and frequent comorbidity requiring a complex array of interventions for the older adult and his or her family. Multiple studies have shown that the first mental health contact for older adults is often a psychiatric hospitalization; hence, psychological treatment for older adults living in the community could help to reduce the severity of disorders and thus reduce the amount and cost of later medical care (Smyer \& Qualls, 1999).

More recently, Kennedy and Tanenbaum (2000) reviewed the literature for evidence of the efficacy of psychosocial interventions with older adults. They noted that although most studies had limitations (such as being conducted in academic settings with self-selected participants who were relatively independent), results from the studies indicated that age-related adaptations of interventions might be necessary with older adults to optimize outcomes. These adaptations include special consideration for any medical comorbidity, regard for the older adult's cognitive capacity, and recognition of the role of family members and caregivers. Therapeutic goals may be modified to include increased selfreliance, reduced primary-care-service needs, improved social or family interaction, and long-term health care planning. Kennedy and Tanenbaum concluded that short-term, focused psychotherapy interventions directed at clinical problems and treatment goals are especially suited for work with older adults. These findings contrast with the earlier studies summarized by Roth and Fonagy (1996), which suggested that older adults may require longer treatment involvement for successful outcomes.

\section{Studies of Effectiveness of Specific Interventions for Mental Health Disorders}

Roth and Fonagy (1996) provided information about effective interventions with older adults for the following issues: anxiety disorders, depression, sleep disturbances, dementias, and benzodiazepine addiction. In the following sections, their findings are summarized, and we then provide information concerning more recent studies. Rather than discuss only benzodiazepine addiction, however, we include a broader section on substance abuse.

Anxiety disorders. It is difficult to determine the prevalence rates for anxiety disorders among older persons, partly because older persons underreport symptoms and partly because high rates of symptoms are reported in subclinical populations (Smyer \& Qualls, 1999). As many as one third of older adults may experience significant anxiety in relation to their life circumstances at any given point in time, making anxiety disorders among the most prevalent mental health problems in later life. Anxiety frequently coexists with depression in older adults and is associated with such diverse causes as marital or family conflict and major physical illnesses such as cardiac disease; hence, differential diagnosis is difficult.

Roth and Fonagy (1996) noted that the state of research concerning treatment approaches for anxiety disorders in later life is severely insufficient. They reported the results of only one study concerning anxiety disorders, which found short-term cognitive-behavioral interventions to be effective with a small sample of outpatients, some of whom were 
also diagnosed with or showed symptoms of additional disorders such as depression, hypochondriasis, and serious medical conditions. Using the evidence from this single smallscale study, Roth and Fonagy (1996) concluded that evidence exists for successfully treating anxiety in older adults using cognitive-behavioral techniques, which have also been shown to work with younger adults.

Stanley and Novy (2000) reviewed additional research that yielded positive results using cognitive-behavioral interventions to treat older adults diagnosed with anxiety disorders. The techniques used in multiple studies included rational emotive therapy, cognitive interventions, relaxation, and meditation. Although the samples in these studies were small, the treatment durations were short, follow-up evaluation was infrequent and, in all but one study, occurred shortly after the termination of treatment, and methods were very limited, Stanley and Novy identified these studies as a basis for encouragement that cognitive-behavioral interventions can be useful with older adults diagnosed with anxiety disorders.

One long-term study reported by Stanley and Novy (2000) evaluated the treatment of 48 older adults, ages 55 to 81 years, who were diagnosed through semistructured interviews with general anxiety disorder (Stanley, Beck, \& Glassco, 1996). Participants were randomly assigned either to a cognitive-behavioral interventions group (i.e., relaxation training, cognitive therapy, and graduated exposure practice in worry-producing situations) or to a supportive psychotherapy group. Treatment lasted 14 weeks, and 31 of the original 48 participants completed the study. Members of both the cognitive-behavioral intervention group (50\%) and the supportive psychotherapy group (77\%) showed significant improvements and maintained those improvements for at least 6 months. Participant self-report and clinicianrated measures of worry, anxiety, and depression were used to measure improvements. Stanley and Novy (2000) noted that although this study was designed to be a controlled trial for cognitive-behavioral interventions with older adults, the control group was a treatment group that turned out to be successful. Because of the success in the supportive psychotherapy group, they concluded that additional studies were needed to determine whether either of the interventions was responsible for the measured improvements or whether some other factor accounted for treatment success.

Depression. Depression has often been noted to be the most significant and widespread mental health challenge of later life, with major depression affecting as many as $15 \%$ of older persons at any point in time and contributing to high rates of suicide, especially among older White men (Smyer \& Qualls, 1999). Roth and Fonagy (1996) examined outcome research for older adults using individual psychological approaches, group therapies, and bibliotherapy for the treatment of depression. Each approach was successful to some degree with some client groups.

Scogin and McElreath (1994) reviewed 17 comparative studies of psychosocial treatments for depression, published between 1975 and 1990, to determine the efficacy of psychosocial treatments for depression in older adults. Between
16 and 162 older adults participated in each study, and the mean number of participants was 45 . The mean ages of participants ranged from 61.95 to 85.0 years, with an overall mean age of 70.5 years. In some studies, the participants were identified as having subclinical depression, and other studies used only participants who were diagnosed with major depression, but most studies evaluated psychosocial treatments with a mixture of participants who had either subclinical depression or major depression. The mode of therapy varied, with 5 studies using individual treatment, 2 studies using self-administered treatment, and the remaining 10 studies using group treatment. Therapists varied by study and included psychiatrists, psychologists, social workers, and psychology graduate students. Psychosocial treatments were compared with no treatment, pill placebos, attention, and unstructured reminiscence. Some of the different approaches (behavioral, interpersonal, psychodynamic, reminiscence, and supportive) were compared with each other when studies offered direct comparisons.

Scogin and McElreath (1994) used posttreatment scores of experimental and control groups to determine the effects of treatment. Their review yielded an overall effect size of 0.78 , an effect size of 0.85 for seven studies using cognitive therapy, and an effect size of 1.05 for eight studies using reminiscence therapy; all effect sizes were in relation to no treatment or a placebo condition. These effect sizes suggest that psychosocial interventions provide effective treatment for older adults who experience depressive symptoms. Comparisons of different psychosocial approaches did not indicate superiority for any particular psychosocial treatment modality.

The primary outcome research concerning individual psychological approaches used in the treatment of later-life depression reported by Roth and Fonagy (1996) examined the efficacy of behavioral, cognitive, and brief psychodynamic therapies and no treatment in an outpatient population (Thompson, Gallagher, \& Breckenridge, 1987). One hundred-twenty people 60 years of age or older who were diagnosed with major depressive disorder were randomly assigned in equal numbers to four groups - three treatment groups and one delayed-treatment control group. The random assignment was somewhat constrained to create comparable groups based on age, sex, severity of depression, and presence of symptoms. Ninety-one of the participants completed the study. Participants in the treatment groups received 16 to 20 sessions of individual therapy over a 16-week period. In the behavioral approach group, treatment focused on increasing participation in social activities through social reinforcement. In the brief psychodynamic approach group, treatment relied primarily on the therapeutic relationship to develop participant insight into problems and create plans for change. Treatment in the cognitive approach group was not described. Multiple questionnaire-style self-report assessments and therapist ratings were used to diagnose and identify levels of depression, other symptoms, associated cognitive features, and overall adjustment and coping. Videotape reviews and weekly supervision sessions were used to verify that psychologists, who served as therapists, followed 
the assigned treatment modalities. The authors identified a significant treatment effect for each treatment group as compared with the control group. No difference was found between the three treatment groups immediately after treatment or at 1year and 2-year follow-ups. Patient commitment to and involvement in therapy was identified as a key predictor of clinical improvement. The results of this study provide further evidence supporting the use of behavioral, brief psychodynamic, and cognitive treatment approaches for older adults who are diagnosed with major depressive disorder.

Roth and Fonagy (1996) cited two studies that provided evidence supporting the use of group therapies with older adults experiencing depression. In one study, psychodynamic group therapy and cognitive-behavioral group therapy approaches were compared and found to be equally effective in reducing levels of depression in 20 community residents, 55 years of age and older, who were diagnosed with major depressive disorder (Steuer et al., 1984). Participants were assigned to one of four treatment groups led by cotherapists who followed a treatment manual for either psychodynamic group therapy or cognitive-behavioral group therapy. A control group was not included. Comparisons of observer-ratings and self-ratings captured at intervals over the 9-week treatment span indicated that participants from both treatment conditions improved in all measured areas, which included symptoms of depression and anxiety. Although no clinical differences were observed, statistical differences from one measure suggested that symptoms of depression in the cognitive-behavioral group participants may have decreased more than did symptoms of depression in the psychodynamic group participants. No follow-up measures were taken, thus long-term effects of treatment were not evaluated.

In another study, the use of a long-term support group after hospital discharge was found to prevent relapse and reduce the number of patients referred again for treatment and readmitted to the hospital (Ong, Martineau, Lloyd, \& Robbins, 1987). In this study, 20 participants, with a mean age of 70 years, who were diagnosed with depression were randomly assigned to either a support group or a control group. The coleaders of the support group used psychodynamic theory and problem-solving techniques as their main therapeutic approaches. At the start of the 9-month study, all participants were living in the community. Pre- and posttreatment clinician-rated measures and archival data were used to evaluate the effects of the support group. During the 9 months of the study, most of the members of the control group were referred again for treatment or readmitted to the hospital, and one member of the control group attempted suicide, whereas no members of the support group were referred again for treatment or readmitted or attempted suicide. The results suggested that the use of a long-term support group after hospitalization discharge may be effective in reducing relapse of depression in older adults.

Roth and Fonagy (1996) also reported a study that evaluated the efficacy of self-help books with older adults who are mildly and moderately depressed (Scogin, Jamison, \& Gochneaur, 1989). Media announcements were used to re- cruit 67 community-dwelling participants 60 years of age and older who had depressive symptoms. After screening, participants were randomly assigned to a cognitive bibliotherapy group, a behavioral bibliotherapy group, or a delayedtreatment control group. Participants in the cognitive bibliotherapy group received a cognitive therapy self-help book, and participants in the behavioral bibliotherapy group received a behavioral therapy self-help book. They were instructed to read the book provided. Follow-up during the following 4 weeks consisted of telephone calls, lasting approximately 5 minutes, during which researchers answered any questions about the reading material. Participants in the delayed-treatment control group were also contacted weekly for 4 weeks before being assigned to a bibliotherapy group. Data from self-report questionnaires and behavioral observations were used to measure general depression, cognitive features of depression, and behavioral aspects of depression. The results revealed a clinically significant change in levels of depression when either cognitive or behavioral therapy self-help books were used. The 44 participants who completed the study retained these gains at 6-month and 2 year follow-ups.

In summary, a variety of interventions have been demonstrated to be effective in treating older persons diagnosed with subclinical or clinical depression. These include reminiscence; individual behavioral, cognitive, and brief psychodynamic therapies; group psychodynamic and cognitive-behavioral therapies; and self-help bibliotherapy.

Sleep disturbances. Sleep disturbance, including difficulty falling asleep or staying asleep, is common in later life (Bootzin, Epstein, Engle-Friedman, \& Salvio, 1996). Roth and Fonagy (1996) cited a study by Friedman, Bliwise, Yesavage, and Salom (1991) that demonstrated the effectiveness of relaxation methods, in comparison to sleep restriction, in assisting 22 mildly depressed older adults, living in the community, who complained of sleep disturbance. The average age of the participants was 69.7 years, and participation included 2 weeks of baseline reporting, 4 weeks of treatment, and 2 weeks of follow-up. Although they were unable to locate adequate outcome research to substantiate the efficacy of interventions other than those reported in this study, Roth and Fonagy implied that education concerning sleep, sleep clinics, and the reevaluation of sleeping medication that is repeatedly prescribed might be helpful when working with older adults experiencing sleep impairment.

A more recent study compared the use of cognitivebehavioral interventions, relaxation therapy, and a behavioral placebo intervention with 75 adults, ages 40 to 80 years, who experienced chronic primary insomnia (Edinger, Wohlgemuth, Radtke, Marsh, \& Quillian, 2001). Cognitive-behavioral interventions included sleep education, stimulus control, and restrictions on time in bed. The relaxation intervention focused on progressive muscle relaxation, and the placebo intervention was a quasi-desensitization treatment. At the 6-month follow-up, the cognitive-behavioral intervention group showed greater improvements in the majority of the outcome measures than did the behavioral and placebo intervention groups. 
Although the mean age of the participants was relatively young (55.3 years), the results of this study indicate that cognitivebehavioral interventions can be useful for some older adults who experience chronic primary insomnia.

Dementias. The most common cause of dementia in later life is Alzheimer's disease, which affects 4 million persons at present and increases in incidence each decade after age 60 (Alzheimer's Disease Education \& Referral Center, 2000). Despite considerable medical research, there remains no known cause of or cure for this disease, and treatment is limited to pharmacological interventions that slow its progress rather than provide a cure. The brain of the Alzheimer's patient is affected in a progressive manner, resulting in total disability and eventual death. The course of the disease may take as long as 12 to 20 years, during which time the family members become the focus of treatment interventions to assist them in coping with caregiver burden (Smyer \& Qualls, 1999).

Roth and Fonagy (1996) acknowledged numerous difficulties in developing empirical research on outcomes in dementia mental health treatment. For example, it is difficult to obtain control groups for such research, and without a control group, stability of function and slowing of decline are difficult to discern. In addition, the success of interventions varies depending on the stage of the dementia. Instead of limiting the information they provided concerning dementia treatments to empirical outcome research, Roth and Fonagy provided a brief synopsis of the types of research available (or not available) for the following common interventions used with dementia patients: special design of the care environment, reality orientation, reminiscence, validation therapy, psychotherapy, cognitive-behavioral therapy, and behavior modification.

Reality orientation is the most extensively evaluated psychological approach to treating dementia and is used exclusively in long-term care settings (Roth \& Fonagy, 1996). Holden and Woods (1995) reviewed 21 controlled trials that indicated that reality orientation had a small but significant effect on measures of verbal orientation. This effect was in comparison to control groups or unstructured social groups. A few limited studies indicated effectiveness of 24-hour reality orientation in the areas of finding one's way around the ward or home, spatial orientation, cognitive abilities, and behavior. A recent meta-analysis of the reality orientation literature compared the results from six randomized controlled trials (Spector, Davies, Woods, \& Orrell, 2000). All studies that were analyzed had significant results in favor of treatment, but none of the studies provided follow-up data. Significant results were found in the areas of cognitive abilities, memory, and information/orientation.

The results of studies of reminiscence, validation, and similar therapies are essentially the same as the results reported here for reality orientation. These approaches are typically used in inpatient settings and have an effect on cognitive abilities and daily functioning. Unfortunately, the literature is lacking in studies of interventions for older persons with dementia who are living in the community. Most such studies provide treatment for caregivers in an attempt to reduce caregiver burden (e.g., Connell, Janevic, \& Gallant, 2001). The older adult experiencing dementia has traditionally been seen as a recipient of respite care rather than as a client for mental health practice; however, recently an argument has been made that the impaired older adult should be represented in research as well as interventions in order to address the myriad of cognitive, behavioral, and emotional concomitants of dementia (Woods, 2001).

Substance abuse. Substance abuse among older adults is a significant but hidden and underreported phenomenon (Myers, Dice, \& Dew, 2000). Blow (1998) noted that as many as $17 \%$ of older adults may misuse or abuse prescription drugs or alcohol. Accurate assessment is difficult and complicated by comorbidity of dementia and other mental as well as physical disorders, including sleep disorders (Smyer \& Qualls, 1999). Although treatment outcomes tend to be less successful in age-heterogeneous groups, Holian (2000) found that older adults (over age 55 years) who abuse substances could be treated effectively in age-specific groups that emphasized the provision of social support and avoidance of confrontation. Holian noted that modifying treatment to meet the needs of older clients was essential to success.

Blow (1998) coordinated a large-scale meta-analysis of research on alcohol and substance abuse in later life, under the auspices of the Substance Abuse and Mental Health Services Administration (SAMHSA). A national panel of experts was convened to provide treatment recommendations based on cross-disciplinary research that included cognitive-behavioral and group approaches, individual counseling, marital and family therapy, and case management. A major conclusion of this panel was that persons who begin drinking heavily later in life, who presumably began drinking due to stressful life circumstances and losses, have more positive treatment outcomes than do persons who began drinking at an earlier point in life, who have well established patterns of alcohol abuse. At the same time, Blow also noted that research is lacking on gender and ethnic variation, the effects of aging on the course of alcohol abuse and treatment, problems and differential treatment strategies for persons who abuse alcohol earlier and later in life, prevention of abuse, and relapse prevention. Furthermore, he underscored the need for research on stress, coping, adaptation, and the relationship of these factors to substance abuse. Although counselors were included in the membership of the SAMHSA review panel, none of the studies examined in this project were conducted or reported in the literature by professional counselors.

\section{STUDIES OF COUNSELING EFFECTIVENESS FOR OLDER PERSONS: LIFE TRANSITIONS}

The studies described earlier were conducted almost exclusively by mental health professionals other than counselors. Because the counseling profession is grounded in a developmental, wellness orientation (see Locke, Myers, \& Herr, 2001), counselors may be expected to conceptualize client concerns from a nonpathological or wellness orientation. In 
this section, we consider studies of counseling effectiveness for older persons, first in relation to common late-life transitions and then followed by a review of outcome research relative to preferred interventions for older adults.

\section{Counseling for Common Late-Life Transitions}

As mentioned earlier, common late-life transitions include but are not limited to coping with loss, adjusting to retirement and reduced income, grandparenthood, second careers, and creating satisfying leisure lifestyles. Much of the literature addressing these issues explains the dynamics of, and potential problems with, late-life transitions and outlines strategies for intervention; however, most recommended strategies are based in theory rather than empirical findings demonstrating what is effective. In addition, the majority of the studies include persons of younger as well as older ages and focus on the dynamics of particular situations or life circumstances and correlates of adjustment, rather than on how to intervene to reduce the negative impact of life transitions and change. Conclusions from the literature apply in a general way rather than solely to the older population. Brief summaries of the literature on widowhood, caregiving, and grandparenthood provide examples of the types of information available to counselors as a basis for evidence-based practice.

Widowhood. Wells and Kendig (1997) noted that caregiving issues "dominate the gerontological literature, while there has been little recent interest in widowhood" (p. 666). At the same time, they noted that widowhood is the more severe transition, creating significant depression, while caregiving "often seriously threatens the meaningfulness of life" (p. 666). Most research on adaptation to widowhood in later life has focused on women, because women tend to outlive their spouses. Both women and men who are widowed in later life report lower life satisfaction and well-being than do those who remain married, and widowhood is a risk factor for dying, whereas being married is not (Arbuckle \& deVries, 1995).

Most outcome research with bereaved older persons has used unstructured group interventions, frequently led by peers or paraprofessionals, with success reported in reducing depression and increasing feelings of support. For example, Folken (1991) described a national program to help newly widowed persons that uses trained paraprofessional counselors for outreach and support. Follow-up questionnaires completed by 62 participants who attended bimonthly talk groups revealed that most thought that the group experience was positive.

Lund and Caserta (2001) compared two studies of spousal bereavement, including samples of 192 and 339 men over the age of 50 years. For both samples, support groups were beneficial for facilitating the grieving process, and mixedgender groups were especially effective. The authors noted that men in their 50 s were more effective in coping with spousal loss than were men in their 70s.

Caregiving. Increasingly, older persons, especially older women, are placed in caregiving roles, as are adult children of older parents who may themselves be older adults or midlife adults. In fact, an estimated one third or more of caregivers are themselves older persons (AARP, 2000). Noting that "caring for an elderly family member at home may cause an intolerable strain, referred to as caregiver burden" (p. 383), Vrabec (1997) underscored the need to help caregivers cope with the negative concomitants of providing care. She reviewed caregiver burden studies from 1980 to 1995 and concluded that social support is the strongest identified correlate of reduced burden; however, studies of counseling effectiveness in promoting social support were not identified in this review. Ballard, Lowery, Powell, O'Brien, and James (2000) reviewed the literature on dementia and perceived burden and concluded that multiple studies point to the success of cognitive-behavioral interventions in reducing the strain of providing care.

Among approximately 5,000 articles written between 1995 and 2002 that deal with caregivers and their mental health concerns (reviewed by Myers, 2003), only a handful tested the efficacy of counseling interventions. Among the few articles that tested interventions, psychoeducational groups and support groups conducted by health care providers, nurses, or mental health professionals were found to be effective. Professional counselors were included in only one study. Schwiebert and Myers (1994), operating from a counseling perspective, developed a psychoeducational support group intervention for midlife caregivers with parentcare responsibilities. They randomly assigned 51 White caregivers to a treatment or a delayed-treatment control group consisting of four 2-hour sessions. Using published measures of knowledge about aging, caregiver burden, and coping resources, they found that a time-limited, structured intervention was successful in reducing participants' perceptions of burden and increasing their coping responses.

Grandparenthood. The normative experience of "grandparenting" has been studied extensively, including factors such as grandparent roles and styles, relationships between grandparents and their grandchildren, the mediating role of parents in grandparent-grandchild relationships, grandparents raising grandchildren, and to a lesser extent, sexual abuse by grandparents (Thomas, Sperry, \& Yarbrough, 2000). The effects of parental divorce on grandchildren, particularly when adult children remarry and grandparents are denied access to their grandchildren, has also been studied extensively. The meaning of grandparenting has been examined, factors that affect grandparent well-being have been described, and variations in grandparenting roles and the expectations of differing minority groups have been examined. We were unable to find any research that described counseling interventions to assist grandparents in coping with the joys and challenges of grandparenthood and, consequently, could find no outcome studies in this area.

\section{Preferred Counseling Interventions for Older Adults and Outcome Research on Effectiveness}

Aging is a part of the life span rather than being apart from the life span. All persons who age experience common life 
transitions and developmental challenges, notably the search for ego integrity identified by Erikson (Santrock, 2002). The design of interventions to help older persons cope with these normative changes has focused on two primary and overlapping strategies: group counseling and life review therapy. Research on both options reveals almost universal support for their effectiveness in helping older persons to develop successful coping mechanisms for a variety of life problems and also to achieve life satisfaction despite unwanted change.

Group counseling. Group counseling has long been identified as the treatment of choice for most problems and challenges of later life (Aday \& Aday, 1997). Lubin, Wilson, Petren, and Polk (1996) reviewed 1,793 research reports and books on group methods, organized in five age groups, demonstrating the effectiveness of group approaches with older persons. Both topic-specific groups (e.g., those dealing with topics such as widowhood, specific health challenges, adjustment to retirement, and creation of leisure lifestyles) and participant-specific groups (e.g., groups designed for older men, older women, bereaved persons, and older adults dealing with substance abuse) are recommended. Groups conducted in institutional settings and community settings seem to be equally, although not universally, effective. For example, Conrod and Overbury (1998) evaluated 49 older persons (mean age of 70 years) with significant visual impairment to determine the effectiveness of perceptual training, individual counseling, and group counseling in facilitating adjustment to their disability. They found that individual counseling was superior to group methods in facilitating optimal adjustment.

Powers and Wisocki (1997) used a focus group intervention with 21 older persons (mean age of 78.1 years) to examine the effects of the intervention on worry and anxiety. Nine persons did not complete the study, leaving only 13 participants for the final analysis. Examination of pre- and postintervention scores on measures of worry, life satisfaction, and psychological symptoms revealed a reduction in the percentage of each day spent worrying.

Although additional studies of group work with older persons could be reported, the results are consistent with those discussed here. Moreover, as noted previously, the studies that exist have included social workers, psychiatric nurses, psychologists, and paraprofessionals rather than professional counselors. Few outcome studies of group interventions with older persons conducted by professional counselors appear in the literature.

Life review therapy. Because life review is "normal" in later life and necessary for the achievement of ego integrity, life review has emerged as the treatment of choice in virtually all settings in which older adults congregate for voluntary reasons or through institutional or group-living placements (Schwiebert \& Myers, 1994). Birren and Cochran (2001) reported successful results from 25 years of research using autobiography groups as a form of structured life review, noting that the sharing of stories in group settings has positive therapeutic benefits for older adults.

Few studies have evaluated the long-term effectiveness of life review therapy. One notable exception is a study by
Haight, Michel, and Hendrix (2000), who followed 52 participants, ages 70 to 88 , over a 3 -year period to evaluate a structured life review process as a holistic nursing intervention. They assigned participants to two groups to receive either friendly visiting or a life review intervention. The results indicated significant improvement in life satisfaction and self-esteem and decreased incidence of depression in nursing home residents tested at 1,2 , and 3 years postintervention for those who received life review therapy.

Although life review is widely used, the application of this approach is often left to paraprofessionals and persons with little professional counseling training. Myers and Schwiebert (1996) cautioned that life review does not always result in positive outcomes, and as a consequence, it is important for those using life review to be trained to recognize the limits of their competence and to refer clients when necessary for more appropriate interventions. Often such interventions involve treatment for depression, because the negative outcome of the life review/ego integrity process is a sense of despair. In addition, these authors recommended using early recollections (ERs) as an alternative to life review and reported positive results using ERs as a shortterm individual and group technique among older adults living in the community. Only anecdotal reports from selected client records were reported as evidence in support of these recommendations.

\section{DISCUSSION}

Older persons experience a myriad of changes that require adjustment and coping, and most older persons successfully negotiate the challenges of later life. Although contemporary studies reveal that most persons age well, changing life circumstances may combine to create the need for mental health interventions for a significant proportion of the older population at any point in time. Outcome research studies with older persons reveal a lack of available services combined with positive outcomes when mental health services are provided. Most available research documents treatment for diagnosable disorders, with relatively few outcome studies supporting preventive and developmental interventions for common late-life transitions. Moreover, most outcome research has included mental health providers other than professional counselors. The results of this review have implications for professional counseling practice, counselor education and supervision, and research.

\section{Evidence-Based Practice in Gerontological Counseling}

Sufficient evidence exists to support the need for specialized approaches to clinical work with older persons in addition to strategies typically used when counseling persons of any age. Longevity, life circumstances, social supports and resources, and health may differ for older adults and should be considered when developing appropriate interventions targeted to the needs of this population. To provide such services effectively, counselors should be aware of the unique 
aspects of late-life development and the special needs of subgroups of older persons. Abundant research exists that identifies a myriad of special characteristics and needs of older persons; however, limited empirical data are available to help counselors choose effective interventions to address those identified needs.

A major finding from the current literature review is the changing perception of older persons reflected in new research that demonstrates the increasing potential for aging well. Furthermore, the receptiveness of older persons to mental health interventions and their responsiveness to such interventions is reported to be increasingly positive. The fact that older persons respond to interventions as well as or better than persons who are younger suggests the need for counselors to address issues of age bias in order to help overcome the current lack of service to this population.

Although the findings are somewhat equivocal, the preponderance of results of multiple outcome studies suggest that counselors should be prepared to modify treatment plans to accommodate the needs of older clients. Both longer individual sessions and longer periods of intervention may be required for successful outcomes with this population. Kennedy and Tanenbaum's (2000) review is an especially useful resource for counselors, because these authors discuss in detail the modifications that may be required. The important role of family members and caregivers and the need to involve members of family systems in treatment are important considerations with older adult clients.

Prevention and early intervention are critical, given the tremendous complexity of interacting factors that may present in the older client and the high probability of medical comorbidity. This complexity mandates a team approach to intervention, with counselors viewing themselves as part of a multidisciplinary team that evaluates and simultaneously treats components of multiple interacting problems. Dual diagnosis and treatment issues may be even more complex in older adult populations, because issues such as loss and transition can create additional needs for adjustment and coping.

The results of outcome research concerning treatment of psychosis and anxiety disorders in older persons are particularly promising. These studies provide a new and positive perspective on the responsiveness of older persons to helping interventions. Counselors need to be alert for signs of anxiety and to be adept at differential diagnosis of anxiety and depression, given new research suggesting that anxiety is also a prevalent mental health condition presenting in older clients. Previous studies have underscored the prevalence of depression, leading to differing types of interventions that may be less effective with anxiety disorders.

Gatz et al. (1998) evaluated psychosocial interventions for older adults against a set of criteria developed by the Division of Clinical Psychology of the American Psychological Association. Gatz et al. summarized the success of various psychological treatments in terms of being "wellestablished" or "probably efficacious." Well-established interventions included behavioral and environmental treat- ments for behavior problems in persons with dementia. Probably efficacious interventions included cognitive, behavioral, and brief psychodynamic therapies for depression, life review therapy for older persons with depression and older persons living in institutional settings, cognitive-behavioral interventions for sleep disorders, psychoeducational support groups for caregivers, and cognitive retraining for older persons with dementia. Our review supports these conclusions; however, considering the magnitude of the effects reported by Scogin and McElreath (1994), cognitive and life review therapies with effect sizes of .85 and 1.05 , respectively, may actually fall under the heading of "well-established." In addition, our current review of outcome research supports the use of group counseling interventions for both developmental and remedial issues associated with aging and late-life development.

Several authors cited in this review recommended longer term follow-up for older clients than is typical with persons who are younger. In particular, long-term support groups are important for relapse prevention in the treatment of severe disorders requiring inpatient care. Problem solving, bibliotherapy, psychoeducational, and social support groups have proven to be effective interventions across a variety of diagnoses and situations (e.g., bereavement). These approaches can also be effective on an individual basis, and in some instances (e.g., adjustment to vision loss), individual counseling may be superior to group approaches.

A review of the prevalence of various disorders and adjustment issues in later life underscores the importance of assessing these conditions with older clients. Furthermore, counselors may find it useful to normalize such issues as anxiety, depression, and sleep disturbance, given their high incidence in the population of older adults. Outcome studies using relaxation have been associated with positive outcomes for each condition; hence, counselors are advised to include relaxation strategies in their repertoire of skills for helping older adult clients.

The reluctance of older clients to seek counseling is due in part to historical stigma associated with mental health service; due in part to lack of public awareness of mental health services; and due in part to a lack of counselor preparedness, attitudinally or in terms of skills, for work with this population. Counselors seeking to provide services to older persons may need to be active in recruiting clients from community as well as institutional settings. This may require a willingness to talk with groups and individuals and actively advocate for the mental health needs in this special population. The fact that older clients receiving Medicaid or other third party reimbursements may be more open to receiving counseling underscores the need for counselors to be recognized providers with parity among other mental health professions. Again, this is an important advocacy issue, with successful outcomes requiring the formation of coalitions between counselors and other mental health providers (Myers, Sweeney, \& White, 2002). Perhaps we, as counselors, will feel a greater sense of efficacy as coalition participants if we first develop a sound knowledge base in gerontological practice. 


\section{Counselor Education and Supervision Issues in Gerontological Counseling}

More than 25 years ago, Blake (1975) referred to older persons as "the forgotten and ignored" (p. 733) of the counseling profession. Myers (1995) and Myers and Blake (1986) have argued for the need to train counseling professionals to meet the needs of older clients. The success of efforts to develop such training is chronicled in the article "From 'Forgotten and Ignored' to Standards and Certification: Gerontological Counseling Comes of Age" (Myers, 1995). Unfortunately, since 1995, the National Board for Certified Counselors has suspended the National Certified Gerontological Counselor credential due to lack of certificants (counselors certified in the gerontological specialty), and many universities have discontinued specialty courses in gerontological issues due to faculty retirements and lack of interest in teaching such courses on the part of new faculty. If counselors are to graduate with training to meet the needs of older clients, infusion of gerontological issues into counselor preparation courses and curricula will be necessary (Myers, 1995).

Studies of service use and general outcome studies reveal that older persons respond as well as or better than younger persons to a variety of mental health interventions. As a consequence, it is important to examine reasons underlying the current extent of under service to this population. Although studies that include psychologists have been reported in the literature, we were unable to find comparable studies involving counseling practitioners. If specialized training is used to estimate services, then the only possible conclusion is that counselors also underserve the older population. The Council for Accreditation of Counseling and Related Educational Programs (CACREP; 2003) has recently reported only two counselor training programs accredited in gerontological counseling. Lack of knowledge about older clients may indeed be a disincentive to counselor practitioners to offer services to older clients. Again, the need for infusion of gerontological issues into the training of all counselors should be a priority because, given population statistics, it is likely that all counselors will encounter older persons or families of older persons (i.e., caregivers) as clients.

Counselors working in the field who did not obtain training in gerontological issues as part of their professional preparation as well as counseling students are encouraged to attend training sessions at professional association conferences to learn more about the needs of the older population. Counselors are encouraged to pay particular attention to new research that establishes effective practices and to participate in clinical research that contributes to the body of knowledge on outcome research with older populations. Training in methods proven effective with older clients, such as cognitive behavior, life review, and bibliotherapy, is an important part of the gerontological counselor's repertoire, and counselors lacking skills in these interventions may find it useful to attend conferences and workshops to develop both knowledge and skill in the application of these approaches.
In addition to continuing education, counselors may find it useful to seek and participate in supervision, both individual and group, related to practice with older clients. This may be especially important for counselors who lack specialized training in gerontological issues and who encounter few older persons among their clientele. Unfortunately, it may be difficult or even impossible to obtain such supervision from licensed counseling practitioners; hence, counselors need to be willing to seek assistance from other mental health professionals who have more extensive experience working with older clients.

\section{Implications for Research}

The counseling profession has strong roots in a developmental, preventive, wellness philosophy (Locke et al., 2001). As a consequence, most of the literature in the professional counseling field reflects a wellness orientation rather than the medical model predominant in earlier discussions of outcome studies. In contrast to studies reported on diagnosable mental health disorders, which are almost exclusively reported in the literature of other mental health disciplines, a variety of articles related to older persons may be found in the literature in professional counseling. However, there is a paucity of outcome research studies in this literature.

Outcome studies are needed to determine not only between-group variation (i.e., how older persons respond to treatment in contrast to younger persons) but also withingroup variation. In particular, research is lacking on counseling outcomes with older minorities, both cultural minorities and persons representing diversity regarding factors such as sexual orientation, disability, and spirituality. Studies of multigenerational family counseling are needed as a foundation for developing effective intergenerational interventions. Furthermore, studies are needed that test multiple interventions in relation to issues such as family development and change in later life, the transition to grandparenting, coping with widowhood, and retirement.

Given the current lack of counseling services to older persons, combined with a paucity of outcome research with this population, counselors are encouraged to conduct both quantitative and qualitative studies of intervention effectiveness. Single-case designs and case studies can provide important information for case conceptualization and treatment, and pre-post measures gathered over time in clinical practices can provide an important knowledge base for future service with older clients. Sharing of experiences and clinical work between counselors through conferences and publications will contribute to the evidence base for practice in the professional counseling field.

A significant need in the gerontological mental health literature concerns the provision of services from the perspective of different professions and the question of which philosophical approaches to treatment are most effective with which older individuals or groups. For example, the medical model has predominated in treatment design in the past, and the predominant view of professionals and service providers in 
mental health as well as health professions of older persons has also been one reflecting illness and pathology. With a new awareness of healthy aging as the norm until late in life, it is possible that wellness paradigms (e.g., Myers, Sweeney, \& Witmer, 2000) may offer new and potentially effective interventions to meet the needs of a broad array of persons in later life. As is true of other issues addressed in this article, further research is needed to establish the efficacy of wellness interventions with older people. As counselors, we are in a unique position to develop and implement wellness programs and strategies and, in so doing, help to enhance the quality of life for many persons, including ourselves as we continue to age.

\section{REFERENCES}

AARP. (2000). Profile of older Americans. Washington, DC: Author.

Aday, R. H., \& Aday, K. L. (1997). Group work with the elderly: An annotated bibliography. Westport, CT: Greenwood.

Alzheimer's Disease Education \& Referral Center. (2000). Progress report on Alzheimer's disease, 2000. Retrieved August 21, 2003, from http://www.alzheimers.org/pubs/prog00.htm\#Introduction

Arbuckle N. W., \& de Vries, B. (1995). Long-term effects of later life spousal and parental bereavement on personal functioning. The Gerontologist, 35, 637-647.

Ballard, C., Lowery, K., Powell, I., O'Brien, J., \& James, I. (2000). Impact of behavioral psychological symptoms of dementia on caregivers. International Psychogeriatrics, 12, 93-105.

Birren, J. E., \& Cochran, K. N. (2001). Telling the stories of life through guided autobiography groups. Baltimore: Johns Hopkins University Press.

Black, B. S., Rabins, P. V., German, P., McGuire, M., \& Roca, R. (1997). Need and unmet need for mental health care among elderly public housing residents. The Gerontologist, 37, 717-728.

Blake, R. (1975). Counseling in gerontology. The Personnel and Guidance Joumal, 53, 733-737.

Blow, F. C. (1998). Substance abuse among older adults: Treatment improvement protocol (TIP), Series 26. (DHHS Publication No. [SMA] 98-3179) Rockville, MD: Substance Abuse and Mental Health Services Administration.

Bootzin, R. R., Epstein, D., Engle-Friedman, M., \& Salvio, M. (1996). Sleep disturbance. In L. L. Carstensen, B. A. Edelstein, \& L. Dornbrand (Eds.), The practical handbook of clinical gerontology (pp. 398-420). Thousand Oaks, CA: Sage.

Connell, C. M., Janevic, M. R., \& Gallant, M. P. (2001). The costs of caregiving: Impact of dementia on family caregivers. Journal of Geriatric Psychiatry and Neurology, 14, 179-187.

Conrod, B. E., \& Overbury, O. (1998). The effectiveness of perceptual training and psychosocial counseling in adjustment to the loss of vision. Journal of Visual Impairment and Blindness, 92, 464-482.

Conte, H. R. (1998). Book reviews [Review of the book What works for whom? A critical review of psychotherapy research]. American Journal of Psychotherapy, 52, 252-253.

Council for Accreditation of Counseling and Related Educational Programs. (2003). Directory of CACREP-accredited programs. Alexandria, VA: Author.

Council for the National Register of Health Service Providers in Psychology. (1985). National register of health service providers in psychol. $o g y$. Washington, DC: Author

Edinger, J. D., Wohlgemuth, W. K., Radtke, R. A., Marsh, G. R., \& Quillian, R. E. (2001). Cognitive behavioral therapy for treatment of chronic primary insomnia: A randomized controlled trial. JAMA, The Journal of the American Medical Association, 285, 1856-1864.

Folken, M. (1991). The importance of group support for widowed persons. Joumal for Specialists in Group Work, 16, 172-177.
Friedman, L., Bliwise, D. L., Yesavage, J. A., \& Salom, S. R. (1991). A preliminary study comparing sleep restriction and relaxation treatments for insomnia in older adults. Joumal of Gerontology, 46, 1-8.

Gatz, M., Fiske, A., Fox, L. S., Kaskie, B., Kasl-Godley, J. E., McCallum, T. J., et al. (1998). Empirically validated treatments for older adults. Journal of Mental Health and Aging, 4, 9-46.

Haight, B. K., Michel, Y., \& Hendrix, S. (2000). The extended effects of the life review in nursing home residents. International Journal of Aging and Human Development, 50, 151-168.

Holden, U. P., \& Woods, R. T. (1995). Positive approaches to dementia care (3rd ed.). Edinburgh, Scotland: Churchill Livingstone.

Holian, M. (2000). Comprehensive treatment program design for older adults with late onset substance abuse (Doctoral dissertation, Miami Institute of Psychology of the Caribbean Center for Advanced Studies, US). Dissertation Abstracts International, 61 (5B), 2763.

Kennedy, G. J., \& Tanenbaum, S. (2000). Psychotherapy with older adults. American Joumal of Psychotherapy, 54, 386-407.

Locke, D., Myers, J. E., \& Herr, E. H. (Eds.). (2001). The handbook of counseling. Thousand Oaks, CA: Sage.

Lubin, B., Wilson, C. D., Petren, S., \& Polk, A. (1996). Research on group treatment methods: Selectively annotated bibliography. Westport, CT: Greenwood Publishing.

Lund, D. A., \& Caserta, M. S. (2001). When the unexpected happens: Husbands coping with the deaths of their wives. In D. A. Lund (Ed.), Men coping with grief: Death, value, and meaning series (pp. 147-167). Amityville, NY: Baywood.

Myers, J. E. (1995). From "forgotten and ignored" to standards and certification: Gerontological counseling comes of age. Journal of Counseling \& Development, 74, 143-149.

Myers, J. E. (2003). Coping with caregiving stress: A wellness-oriented, strengths-based approach for family counselors. The Family Joumal, 11, 1-9.

Myers, J. E., \& Blake, R. (1986). Professional preparation of gerontological counselors: Issues and guidelines. Counselor Education and Supervision, 26, 137-145.

Myers, J. E., Dice, C. E., \& Dew, B. J. (2000). Alcohol abuse in later life: Issues and interventions for counselors. Adultspan, 2, 2-14.

Myers, J. E., \& Schwiebert, V. (1996). Competencies for gerontological counselors. Alexandria, VA: American Counseling Association.

Myers, J. E., Sweeney, T. J., \& White, V. A. (2002). Advocacy for counselors and counseling: A professional imperative. Joumal of Counseling \& Development, 80, 394-402.

Myers, J. E., Sweeney, T. J., \& Witmer, J. M. (2000). The Wheel of Wellness counseling for wellness: A holistic model for treatment planning. Journal of Counseling \& Development, 78, 251-266.

Nordhus, I. H., \& VandenBos, G. R. (Eds.). (1998). Clinical gero-psychology. Washington, DC: American Psychological Association.

Ong, Y., Martineau, F., Lloyd, C., \& Robbins, I. (1987). A support group for the depressed elderly. International Joumal of Geriatric Psychiatry, 2, 119-123.

Powers, C. B., \& Wisocki, P. A. (1997). An examination of the therapeutic effects of focus groups on elderly worriers. International Journal of Aging and Human Development, 45, 159-167.

Roth, A., \& Fonagy, P. (1996). What works for whom? A critical review of psychotherapy research. New York: Guilford Press.

Santrock. J. (2002). Life-span development. New York: McGraw Hill.

Schwiebert, V. L., \& Myers, J. E. (1994). Midlife care givers: Effectiveness of a psychoeducational intervention for midlife adults with parent-care responsibilities. Journal of Counseling \& Development, $72,627-632$.

Scogin, F., Jamison, C., \& Gochneaur, K. (1989). Comparative efficacy of cognitive and behavioral bibliotherapy for mildly and moderately depressed older adults. Joumal of Consulting and Clinical Psychology, 57, 403-407.

Scogin, F., \& McElreath, L. (1994). Efficacy of psychosocial treatments for geriatric depression: A quantitative review. Joumal of Consulting and Clinical Psychology, 62, 69-74.

Smyer, M. A., \& Qualls, S. H. (1999). Aging and mental health. Malden, MA: Blackwell 
Spector, A., Davies, S., Woods, B., \& Orrell, M. (2000). Reality orientation for dementia: A systematic review of the evidence of effectiveness from randomized controlled trials. The Gerontologist, 40, 206-212.

Stanley, M. A., Beck, J. G., \& Glassco, J. D. (1996). Treatment of generalized anxiety in older adults: A preliminary comparison of cognitive-behavioral and supportive approaches. Behavior Therapy, 27, 565-581.

Stanley, M. A., \& Novy, D. M. (2000). Cognitive-behavior therapy for generalized anxiety in late life: An evaluative overview. Journal of Anxiety Disorders, 14, 191-207.

Steuer, J., Mintz, J., Hammen, C. L., Hill, M. A., Jarvik, L. F., McCarley, T., et al. (1984). Cognitive-behavioral and psychodynamic group psychotherapy in treatment of geriatric depression. Journal of Consulting and Clinical Psychology, 52, 180-189.
Thomas, J. L., Sperry, L., \& Yarbrough, M. S. (2000). Grandparents as parents: Research findings and policy recommendations. Child Psy. chiatry and Human Development, 31, 3-22.

Thompson, L. W., Gallagher, D., \& Breckenridge, J. S. (1987). Comparative effectiveness of psychotherapies for depressed elders. Joumal of Consulting and Clinical Psychology, 55, 385-390.

Vrabec, N. J. (1997). Literature review of social support and caregiver burden, 1980 to 1995. Joumal of Nursing Scholarship, 29, 383-388.

Wells, Y. D., \& Kendig, H. L. (1997). Health and well-being of spouse caregivers and the widowed. The Gerontologist, 37, 666-674.

Woods, R. T. (2001). Discovering the person with Alzheimer's disease: Cognitive, emotional, and behavioral aspects. Aging and Mental Health, 5, S7-S16. 